\title{
Three-space problems and bounded approximation properties
}

\author{
by
}

\author{
WolfGang Lusky (Paderborn)
}

Dedicated to Prof. Olek Petczyński on the occasion of his 70th birthday

\begin{abstract}
Let $\left\{R_{n}\right\}_{n=1}^{\infty}$ be a commuting approximating sequence of the Banach space $X$ leaving the closed subspace $A \subset X$ invariant. Then we prove three-space results of the following kind: If the operators $R_{n}$ induce basis projections on $X / A$, and $X$ or $A$ is an $\mathcal{L}_{p}$-space, then both $X$ and $A$ have bases. We apply these results to show that the spaces $C_{\Lambda}=\overline{\operatorname{span}}\left\{z^{k}: k \in \Lambda\right\} \subset C(\mathbb{T})$ and $L_{\Lambda}=\overline{\operatorname{span}}\left\{z^{k}: k \in \Lambda\right\} \subset L_{1}(\mathbb{T})$ have bases whenever $\Lambda \subset \mathbb{Z}$ and $\mathbb{Z} \backslash \Lambda$ is a Sidon set.
\end{abstract}

1. Introduction. Let $X$ be a separable Banach space (over $\mathbb{R}$ or $\mathbb{C}$ ), $A \subset X$ a closed subspace and $(\mathrm{P})$ a Banach space property. Then the paper deals with three-space problems of the following kind:

If $X / A$ satisfies (P), do $X$ and $A$ also have (P)?

Let $B \subset X$ be another closed subspace such that $X=\overline{A+B}$. If $B$ satisfies (P), do $A$ and $X$ also have $(\mathrm{P})$ ?

It turns out that these questions are meaningful if $(\mathrm{P})$ is a bounded ap-

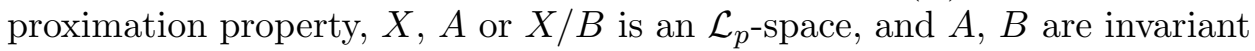
under a sequence of finite rank operators which approximate the identity on $X$. We obtain basis and FDD existence theorems supplementing the results of [9]. In Section 3 we apply these methods to $C_{\Lambda^{-}}$and $L_{\Lambda^{-}}$-spaces (over $\mathbb{T}$ ) and show that $C_{\Lambda}$ and $L_{\Lambda}$ have bases whenever $\Lambda \subset \mathbb{Z}$ is a co-Sidon set.

First we recall some basic definitions. $X$ is called an $\mathcal{L}_{p^{-}}$space $\left(\right.$or $\mathcal{L}_{p, \lambda^{-}}$ space) if there exists a $\lambda \geq 1$ such that, for every finite-dimensional $E \subset X$, there is a finite-dimensional subspace $F \subset X$ with $E \subset F$ and $d\left(F, l_{p}^{\operatorname{dim} F}\right) \leq \lambda$. $(d(\cdot, \cdot)$ is the Banach-Mazur distance.) It is known $([6])$ that in this situation we can even find such $F$ which are uniformly complemented in $X$.

$X$ has the bounded approximation property (BAP) if there is a sequence of bounded linear finite rank operators $R_{n}: X \rightarrow X$ with $\lim _{n} R_{n} x=x$ for all $x \in X ;\left\{R_{n}\right\}_{n=1}^{\infty}$ is then called an approximating sequence (a.s.).

2000 Mathematics Subject Classification: 46B15, 46B03, 46B20. 
If in addition $R_{n} R_{m}=R_{\min (n, m)}$ for $n \neq m$ then $\left\{R_{n}\right\}_{n=1}^{\infty}$ is called a commuting approximating sequence (c.a.s.) and $X$ is said to have the commuting bounded approximation property (CBAP).

$X$ has a finite-dimensional Schauder decomposition (FDD) if there is a c.a.s. $\left\{R_{n}\right\}_{n=1}^{\infty}$ of $X$ where all $R_{n}$ are projections. (In this case we have $X=\sum_{n} \oplus\left(R_{n+1}-R_{n}\right) X$. $)$

Finally, $X$ has a basis provided that $X$ has a c.a.s. $\left\{R_{n}\right\}_{n=1}^{\infty}$ consisting of projections such that $\operatorname{dim}\left(R_{n+1}-R_{n}\right) X=1$ for all $n$.

It is clear that basis $\Rightarrow \mathrm{FDD} \Rightarrow \mathrm{CBAP} \Rightarrow \mathrm{BAP}$. On the other hand it is well known that $\mathrm{CBAP} \nRightarrow \mathrm{FDD} \nRightarrow$ basis $([1],[11],[12]$; see also $[10])$.

In the following, " $"$ means "is isomorphic to". If $U_{n}: X \rightarrow X$, $n=1,2, \ldots$, are linear operators we always put $U_{0}=U_{-1}=\ldots=0$.

We say that the $U_{n}$ factor uniformly through an $\mathcal{L}_{p^{-}}$-space $Y$ if there are linear operators $T_{n}: X \rightarrow Y$ and $S_{n}: Y \rightarrow X$ with $S_{n} T_{n}=U_{n}$ and $\sup _{n}\left\|S_{n}\right\| \cdot\left\|T_{n}\right\|<\infty$.

2. The main results. Again, assume that $X$ is a separable Banach space. Let $A \subset X$ and $B \subset X$ be closed subspaces. Recall that a linear operator $R: X \rightarrow X$ with $R A \subset A$ induces a linear operator $\widehat{R}$ on $X / A$ with $\|\widehat{R}\| \leq\|R\|$, namely $\widehat{R}(x+A)=R x+A, x \in X$.

2.1. TheOREM. Let $\left\{R_{n}\right\}_{n=1}^{\infty}$ be a c.a.s. of $X$ with $R_{n} A \subset A, n=1,2, \ldots$

(a) Assume that the operators $R_{n}$ induce the projections of a basis (or $F D D$, resp.) on $X / A$. If $X$ or $A$ is an $\mathcal{L}_{p}$-space for some $p \in[1, \infty[$ then $X \oplus l_{p}$ has a basis (or an FDD, resp.) with projections $P_{n}$ which leave $A \oplus l_{p}$ invariant. In particular, $A \oplus l_{p}$ also has a basis (or an FDD, resp.) with projections $\left.P_{n}\right|_{A \oplus l_{p}}$.

(b) Assume that $X=\overline{A+B}$ and that $\left.R_{n}\right|_{B}, n=1,2, \ldots$, are the projections of a basis (or an FDD, resp.) of $B$. If $X, X / B$ or $A$ is an $\mathcal{L}_{p}$-space for some $p \in\left[1, \infty\left[\right.\right.$ then $X \oplus l_{p}$ has a basis (or an $F D D$, resp.) with projections $P_{n}$ satisfying $P_{n}\left(A \oplus l_{p}\right) \subset A \oplus l_{p}$ and $\left.P_{n}\right|_{B}=\left.R_{n}\right|_{B}, n=1,2, \ldots$ In particular, $A \oplus l_{p}$ has a basis (or an FDD, resp.) with projections $\left.P_{n}\right|_{A \oplus l_{p}}$.

We postpone the proof of 2.1 to Section 4 . Here we make a few remarks.

REMARKs. The proof of 2.1 shows that the theorem remains true for $p=\infty$. Here we have to replace $l_{p}$ by $c_{0}$.

In 2.1(b) we do not require $A \cap B=\{0\}$. Moreover, we can admit the case that $\left.R_{n}\right|_{B}=\left.R_{n+1}\right|_{B}$ for some $n$. On the other hand, we do not claim that the $\left.R_{n}\right|_{A}$ themselves are the projections of a basis or FDD of $A$. The theorem is certainly false if we drop the assumption that $X, A$ or $X / B$ is an $\mathcal{L}_{p^{-}}$-space (e.g. take $B=\{0\}$ and $A=X$ ). 
In some cases one obtains slightly better results. Then we do not need to add $l_{p}$ or $c_{0}$ :

2.2. Theorem. Let $\left\{R_{n}\right\}_{n=1}^{\infty}$ be a c.a.s. of $X$ which leaves $A$ invariant and defines a sequence of projections for a basis of $X / A$. If $X$ or $A$ is an $\mathcal{L}_{p}$-space for some $p \in[1, \infty]$ then both $X$ and $A$ have bases.

Proof. $\left\{\left.R_{n}\right|_{A}\right\}_{n=1}^{\infty}$ is a c.a.s. of $A$. We claim that $R_{n}-R_{n-1}$ and $\left.\left(R_{n}-R_{n-1}\right)\right|_{A}$ factor uniformly through an $\mathcal{L}_{p}$-space. Indeed, by our assumption, $A \cap\left(R_{n}-R_{n-1}\right) X$ is at most 1-codimensional in $\left(R_{n}-R_{n-1}\right) X$. Hence we find uniformly bounded projections $P_{n}:\left(R_{n}-R_{n-1}\right) X \rightarrow A \cap$ $\left(R_{n}-R_{n-1}\right) X$.

If $X$ is an $\mathcal{L}_{p^{-}}$-space then define

$$
\begin{aligned}
& T_{n}: A \rightarrow X \quad \text { by } \quad T_{n} a=\left(R_{n+1}-R_{n-2}\right) a, \quad a \in A \text {, } \\
& S_{n}: X \rightarrow A \quad \text { by } \quad S_{n} x=P_{n}\left(R_{n}-R_{n-1}\right) x, x \in X \text {. }
\end{aligned}
$$

We obtain $S_{n} T_{n}=\left.\left(R_{n}-R_{n-1}\right)\right|_{A}$. Hence the operators $\left.\left(R_{n}-R_{n-1}\right)\right|_{A}$ factor uniformly through $X$. By [8], $A$ has a basis.

If $A$ is an $\mathcal{L}_{p}$-space then set $W=\left(\right.$ id $\left.-P_{n}\right)\left(R_{n}-R_{n-1}\right) X$ and define $T_{n}: X \rightarrow A \oplus W$ by

$$
T_{n} x=\left(P_{n}\left(R_{n}-R_{n-1}\right) x,\left(\mathrm{id}-P_{n}\right)\left(R_{n}-R_{n-1}\right) x\right),
$$

and $S_{n}: A \oplus W \rightarrow X$ by

$$
S_{n}(a, w)=\left(R_{n+1}-R_{n-2}\right) a+w .
$$

Here $S_{n} T_{n}=R_{n}-R_{n-1}$ and $R_{n}-R_{n-1}$ factors uniformly through $A \oplus W$. The latter space is an $\mathcal{L}_{p, \lambda}$-space (where $\lambda$ does not depend on $n$ ) because $\operatorname{dim} W \leq 1$. Hence $X$ has a basis (in view of [8]).

This proves 2.2 , since separable $\mathcal{L}_{p}$-spaces always have bases ([4]).

In the case $p=1$ and $X$ an $\mathcal{L}_{1}$-space Theorem 2.1(a) can be proved under the considerably weaker assumption that $\left\{R_{n}\right\}_{n=1}^{\infty}$ be an approximating sequence. Similarly the basis version of 2.1 for $p=\infty$ can also be inferred under this assumption.

2.3. Theorem. Let $\left\{R_{n}\right\}_{n=1}^{\infty}$ be an a.s. of $X$ with $R_{n} A \subset A, n=$ $1,2, \ldots$ Assume that the operators $R_{n}$ induce the projections of a basis (or an FDD, resp.) on $X / A$. If $X$ is an $\mathcal{L}_{1}$-space then $X \oplus l_{1}$ has a basis (or an $F D D$, resp.) with projections $P_{n}$ which leave $A \oplus l_{1}$ invariant. In particular, $A \oplus l_{1}$ also has a basis (or an FDD, resp.) with projections $\left.P_{n}\right|_{A \oplus l_{1}}$.

2.4. Theorem. Let $X$ be an $\mathcal{L}_{\infty}$-space and let $\left\{R_{n}\right\}_{n=1}^{\infty}$ be an a.s. of $X$ with $R_{n} A \subset A$ for all $n$. Assume that the $R_{n}, n=1,2, \ldots$, induce the projections of a basis of $X / A$. Then $X \oplus c_{0}$ has a basis with projections $P_{n}$ satisfying $P_{n}\left(A \oplus c_{0}\right) \subset A \oplus c_{0}, n=1,2, \ldots$ In particular, $A \oplus c_{0}$ has a basis with projections $\left.P_{n}\right|_{A \oplus c_{0}}$. Finally, there is a subspace $B \sim c_{0}$ of $X \oplus c_{0}$ such 
that $\overline{\left(A \oplus c_{0}\right)+B}=X \oplus c_{0}$ and the operators $\left.P_{n}\right|_{B}$ are the basis projections of the unit vector basis of $c_{0}$.

We also postpone the proofs of Theorems 2.3 and 2.4 to Section 4 .

Recall that $A \oplus l_{p} \sim A$ provided that $A$ contains a complemented isomorphic copy of $l_{p}$, and $A \oplus c_{0} \sim A$ provided that $A$ contains an isomorphic copy of $c_{0}$ (see [7]). Together with 2.1 and the remark following it we obtain

2.5. Corollary. Let $\left\{R_{n}\right\}_{n=1}^{\infty}$ be a c.a.s. of $X$ and let $A \subset X$ be an $\mathcal{L}_{p^{-}}$ space for some $p \in[1, \infty]$ such that $R_{n}\left(\mathrm{id}-R_{n}\right) X \subset A, n=1,2, \ldots$ Then $X \oplus l_{p}$, if $p<\infty$, and $X \oplus c_{0}$, if $p=\infty$, has an FDD.

Reformulating the basis version of 2.1(b) (with $A=X$ ) we obtain the following basis extension result.

2.6. Corollary. Let $B \subset X$ be a closed subspace with a basis $\Omega$ and assume that $X$ or $X / B$ is an $\mathcal{L}_{p}$-space. If the basis projections of $\Omega$ can be extended to a c.a.s. of $X$ then $X \oplus l_{p}$, for $1 \leq p<\infty$, and $X \oplus c_{0}$, for $p=\infty$, has a basis which contains $\Omega$ as a subsequence.

Remarks. Here we identify $x \in X$ with $(x, 0) \in X \oplus l_{p}$. Note that $\Omega$ is not just equivalent to a subsequence but the elements of $\Omega$ coincide with some elements of the extended basis.

Theorem 2.3 also includes a result of [9]. Recall that every separable Banach space $Y$ is isomorphic to a quotient space of $l_{1}$.

2.7. Corollary. Let $Y$ be a Banach space with basis and let $q: l_{1} \rightarrow Y$ be a quotient map. Then $\operatorname{ker} q$ has a basis.

Proof. Let $\widehat{R}_{n}: Y \rightarrow Y$ be the basis projections of a given basis of $Y$. Moreover, let $\left\{e_{k}\right\}_{k=1}^{\infty}$ be the unit vector basis of $l_{1}$. Find $y_{j} \in Y$ with $\left\|y_{j}\right\|=1$ and integers $0<m_{1}<m_{2}<\ldots$ such that $y_{j} \in \widehat{R}_{n} Y, j \leq m_{n}$, satisfying the following:

For each $y \in \widehat{R}_{n} Y$ with $\|y\|=1$ there are $\lambda_{1}, \ldots, \lambda_{m_{n}}$ such that

$$
y=\sum_{j=1}^{m_{n}} \lambda_{j} y_{j}, \quad \sum_{j=1}^{m_{n}}\left|\lambda_{j}\right| \leq 2 .
$$

Then define the quotient map $q_{0}: l_{1} \rightarrow Y$ by $q_{0} e_{j}=y_{j}$ for all $j$. It is well known $([7])$ that $\operatorname{ker} q_{0} \sim \operatorname{ker} q$. Put $A=\operatorname{ker} q_{0}$. We can assume that $\operatorname{dim} A=\infty$, hence $A \sim A \oplus l_{1}([7])$.

Define the linear operators $R_{n}: l_{1} \rightarrow l_{1}$ by $R_{n} e_{j}=e_{j}$ for $j \leq m_{n}$. If $k>m_{n}$ find $\lambda_{1}, \ldots, \lambda_{m_{n}}$ with 


$$
\widehat{R}_{n} y_{k}=\left\|\widehat{R}_{n}\right\| \sum_{j=1}^{m_{n}} \lambda_{j} y_{j}, \quad \sum_{j=1}^{m_{n}}\left|\lambda_{j}\right| \leq 2 .
$$

Put $R_{n} e_{k}=\left\|\widehat{R}_{n}\right\| \sum_{j=1}^{m_{n}} \lambda_{j} e_{j}$. Then we obtain an a.s. $\left\{R_{n}\right\}_{n=1}^{\infty}$ with $q_{0} R_{n}=$ $\widehat{R}_{n} q_{0}$ for all $n$. Now Theorem 2.3 completes the proof.

3. Co-Sidon sets. Now we turn to complex Banach spaces. Let $\mathbb{T}=$ $\{z \in \mathbb{C}:|z|=1\}$. Fix $\Lambda \subset \mathbb{Z}$ and let $C_{\Lambda}$ be the closed linear span of the functions $z^{k}, k \in \Lambda$, on $\mathbb{T}$ with respect to the sup-norm (denoted by $\|\cdot\|_{\infty}$ ). Moreover, let $L_{\Lambda}$ be the closed linear span of $z^{k}, k \in \Lambda$, with respect to the $L_{1}$-norm on $\mathbb{T}$ (denoted by $\left.\|\cdot\|_{1}\right)$.

We make use of some classical finite rank operators. Fix $n$ and put

$$
\sigma_{n}\left(\sum_{k} \alpha_{k} z^{k}\right)=\sum_{k=-n}^{n} \frac{n-|k|}{n} \alpha_{k} z^{k} .
$$

It is well known $([3])$ that $\left\|\sigma_{n}\right\|=1$ on $C(\mathbb{T})=C_{\mathbb{Z}}$ as well as on $L_{1}(\mathbb{T})=L_{\mathbb{Z}}$. Clearly, $\sigma_{n}\left(C_{\Lambda}\right) \subset C_{\Lambda}$ and $\sigma_{n}\left(L_{\Lambda}\right) \subset L_{\Lambda}$ for each $\Lambda \subset \mathbb{Z}$. For $0<m<n$ put

$$
V_{n, m}=\frac{n \sigma_{n}-m \sigma_{m}}{n-m} \text {. }
$$

Then

$$
V_{n, m}\left(\sum_{k} \alpha_{k} z^{k}\right)=\sum_{|k| \leq m} \alpha_{k} z^{k}+\sum_{m<|k| \leq n} \frac{n-|k|}{n-m} \alpha_{k} z^{k}
$$

and $\left\|V_{n, m}\right\| \leq(n+m) /(n-m)$ (as an operator on $C(\mathbb{T})$ as well as on $\left.L_{1}(\mathbb{T})\right)$.

$\Lambda \subset \mathbb{Z}$ is called a Sidon set if $\left\{z^{k}\right\}_{k \in \Lambda}$ (regarded as a sequence in $\left.C(\mathbb{T})\right)$ is equivalent to the unit vector basis of $l_{1}$. It is well known ([2]) that lacunary sets are Sidon sets and finite unions of Sidon sets are Sidon sets.

3.2. Theorem. Let $\Lambda \subset \mathbb{Z}$ be such that $\mathbb{Z} \backslash \Lambda$ is a Sidon set. Then $C_{\Lambda}$ and $L_{\Lambda}$ have a basis.

Further results on $C_{\Lambda}$ and $L_{\Lambda}$, where $\mathbb{Z} \backslash \Lambda$ is a Sidon set, can be found in $[5]$.

For the proof of 3.2 we need the following

3.3. Lemma. Let $\Lambda \subset \mathbb{Z}$ be such that $\mathbb{Z} \backslash \Lambda$ is a Sidon set. Then $C_{\Lambda}$ contains an isomorphic copy of $c_{0}$ and hence $C_{\Lambda} \sim C_{\Lambda} \oplus c_{0}$. Moreover, $L_{\Lambda}$ contains a complemented copy of $l_{1}$ and $L_{\Lambda} \sim L_{\Lambda} \oplus l_{1}$.

Proof. It is well known $([2])$ that $C(\mathbb{T}) / C_{\Lambda}$ is isomorphic to $l_{2}$ since $\mathbb{Z} \backslash \Lambda$ is a Sidon set. Now find $e_{n} \in C(\mathbb{T})$ of norm one with mutually disjoint supports, which implies that $\left\{e_{n}\right\}_{n=1}^{\infty}$ is equivalent to the unit vector basis of $c_{0}$. Let $q: C(\mathbb{T}) \rightarrow C(\mathbb{T}) / C_{\Lambda}$ be the quotient map. Then 
we must have $\lim _{n}\left\|q e_{n}\right\|_{\infty}=0$ because otherwise we could find a subsequence $\left\{e_{n_{k}}\right\}_{k=1}^{\infty}$ such that $\left.q\right|_{\operatorname{span}\left\{e_{n_{k}}\right\}_{k=1}^{\infty}}$ is an isomorphism, which is impossible since $q C(\mathbb{T}) \sim l_{2}$. So we find $\widetilde{e}_{n} \in C_{\Lambda}$ with $\lim _{n}\left\|e_{n}-\widetilde{e}_{n}\right\|_{\infty}=0$ and hence a subsequence $\left\{\widetilde{e}_{n_{m}}\right\}_{m=1}^{\infty}$ which is equivalent to $\left\{e_{n_{m}}\right\}_{m=1}^{\infty}$. Thus $\overline{\operatorname{span}}\left\{\widetilde{e}_{n_{m}}\right\}_{m=1}^{\infty} \subset C_{\Lambda}$ is isomorphic to $c_{0}$.

It is well known $([2])$ that $L_{1}(\mathbb{T}) / L_{\Lambda}$ is isomorphic to $c_{0}$ since $\mathbb{Z} \backslash \Lambda$ is a Sidon set. Take $f_{n} \in L_{1}(\mathbb{T})$ of norm one with disjoint supports. Then $\left\{f_{n}\right\}_{n=1}^{\infty}$ is equivalent to the unit vector basis of $l_{1}$ and $\overline{\operatorname{span}}\left\{f_{n}\right\}_{n=1}^{\infty}$ is complemented in $L_{1}(\mathbb{T})$. Let $q_{1}: L_{1}(\mathbb{T}) \rightarrow L_{1}(\mathbb{T}) / L_{\Lambda}$ be the corresponding quotient map. Since we are in $c_{0}$ we find a subsequence $\left\{q_{1} f_{n_{k}}\right\}_{k=1}^{\infty}$ of $\left\{q_{1} f_{n}\right\}_{n=1}^{\infty}$ such that

$$
\text { weak- } \lim _{k \rightarrow \infty} q_{1}\left(f_{n_{2 k+1}}-f_{n_{2 k}}\right)=0 .
$$

By Mazur's theorem there are $m_{1}<m_{2}<\ldots$ and suitable convex combinations $g_{k}=\sum_{j=m_{k}+1}^{m_{k+1}} \lambda_{k, j}\left(f_{n_{2 j+1}}-f_{n_{2 j}}\right)$ such that $\lim _{k \rightarrow \infty}\left\|q_{1} g_{k}\right\|=0$. So we find $\widetilde{g}_{k} \in L_{\Lambda}$ with $\lim _{k \rightarrow \infty}\left\|g_{k}-\widetilde{g}_{k}\right\|_{1}=0$. The elements $g_{k}, k=1,2, \ldots$, have disjoint supports and are equivalent to the unit vector basis of $l_{1}$. By going over to a suitable subsequence $\left\{g_{k_{j}}\right\}_{j=1}^{\infty}$ we see that $\overline{\operatorname{span}}\left\{g_{k_{j}}\right\}_{j=1}^{\infty}$, and then also $\overline{\operatorname{span}}\left\{\widetilde{g}_{k_{j}}\right\}_{j=1}^{\infty} \subset L_{\Lambda}$, is isomorphic to $l_{1}$ and complemented in $L_{1}(\mathbb{T})$.

Proof of Theorem 3.2. Put $\Omega=\mathbb{Z} \backslash \Lambda$. Fix an integer $n>0$ and take $m$ with $0<m<n$ such that $(n+m) /(n-m) \leq 2$. Consider

$$
V_{n, m}\left(\sum_{k} \alpha_{k} z^{k}\right)=\sum_{|k| \leq m} \alpha_{k} z^{k}+\sum_{m<|k| \leq n} \frac{n-|k|}{n-m} \alpha_{k} z^{k} .
$$

For $h \in L_{1}(\mathbb{T})$ we define $\mu_{h}: C(\mathbb{T}) \rightarrow \mathbb{C}$ by

$$
\mu_{h}(f)=\frac{1}{2 \pi} \int_{0}^{2 \pi} h\left(e^{-i t}\right) f\left(e^{i t}\right) d t, \quad f \in C(\mathbb{T}) .
$$

Then $\left\|\mu_{h}\right\|=\|h\|_{1}$.

Regard $g_{n}:=\sum_{m<|k| \leq n} z^{k}$ as an element of $L_{1}(\mathbb{T})$. Since $\Omega$ is a Sidon set we obtain a constant $c>0$ independent of $n$ with $\left|\mu_{g_{n}}(f)\right| \leq c\|f\|_{\infty}$ for every $f \in C_{\Omega}$. Put

$$
M_{\Lambda}=\left\{\mu \in C(\mathbb{T})^{*}: \widehat{\mu}(k)=0 \text { if } k \in \mathbb{Z} \backslash \Lambda\right\},
$$

where $\widehat{\mu}(k)=\mu\left(z^{-k}\right)$. Then $C_{\Omega}^{*}=C(\mathbb{T})^{*} / M_{\Lambda}$. Hence we find $\mu \in M_{\Lambda}$ with $\left\|\mu+\mu_{g_{n}}\right\| \leq 2 c$. Consider

$$
\nu:=\left(\mu+\mu_{g_{n}}\right) \circ\left(V_{2 n, n}-V_{n, m}\right) .
$$

By definition of the $V_{j, k}$ we can find a trigonometric polynomial $h_{n} \in$ $\operatorname{span}\left\{z^{k}: m \leq|k| \leq 2 n\right\}$ such that $\nu=\mu_{h_{n}}$. Moreover, $\left\|h_{n}\right\|_{1} \leq 12 c$. 
Put

$$
P_{\Omega}\left(\sum_{k} \alpha_{k} z^{k}\right)=\sum_{k \in \Omega} \alpha_{k} z^{k}
$$

Since

$$
\mu_{h_{n}}(f)=\mu_{g_{n}}\left(\left(V_{2 n, n}-V_{n, m}\right) f\right)
$$

for all $f \in C_{\Omega}$ we obtain

$$
P_{\Omega} h_{n}=P_{\Omega}\left(V_{2 n, n}-V_{n, m}\right) g_{n}=\sum_{\substack{m<|k| \leq n \\ k \in \Omega}} \frac{|k|-m}{n-m} z^{k} .
$$

Now, define $R_{n} f=V_{n, m} f+h_{n} * f, f \in C(\mathbb{T})$. Then the $R_{n}$ are uniformly bounded finite rank operators. Moreover, $\lim _{n} R_{n} f=f$ for all $f \in C(\mathbb{T})$. On $C_{\Omega}$ we have

$$
R_{n}\left(\sum_{k \in \Omega} \alpha_{k} z^{k}\right)=\sum_{\substack{|k| \leq n \\ k \in \Omega}} \alpha_{k} z^{k}
$$

Hence the $\left.R_{n}\right|_{C_{\Omega}}$ are basis projections. We have $C(\mathbb{T})=\overline{C_{\Omega}+C_{\Lambda}}$. Since $R_{n} C_{\Lambda} \subset C_{\Lambda}$ for all $n$, the operators $R_{n}$ define basis projections on $C(\mathbb{T}) / C_{\Lambda}$. By 2.4 with $A=C_{\Lambda}$ the space $C_{\Lambda} \oplus c_{0}$ has a basis. Now, 3.3 shows that $C_{\Lambda}$ has a basis.

The operators $\left.R_{n}^{*}\right|_{L_{1}(\mathbb{T})}$ define an a.s. on $L_{1}(\mathbb{T})$. (Indeed, $R_{n}^{*} f=R_{n} f$ for any trigonometric polynomial $f$.) The operators $\left.R_{n}^{*}\right|_{L_{\Omega}}$ are basis projections. Since $L_{1}(\mathbb{T})=\overline{L_{\Lambda} \oplus L_{\Omega}}$ the operators $R_{n}^{*}$ define basis projections on $L_{1}(\mathbb{T}) / L_{\Lambda}$. By $2.3, L_{\Lambda} \oplus l_{1}$ has a basis. Now 3.3 concludes the proof.

4. Proofs of the main results. In the following let $X$ be a separable Banach space, and $A \subset X$ and $B \subset X$ closed subspaces such that $X=$ $\overline{A+B}$. Put

$$
W_{p}= \begin{cases}l_{p}, & 1 \leq p<\infty \\ c_{0}, & p=\infty\end{cases}
$$

4.1. Proposition. Let $\left\{R_{n}\right\}_{n=1}^{\infty}$ be an a.s. of $X$ with $R_{n}\left(\mathrm{id}-R_{n}\right) X \subset A$ and $R_{n} A \subset A$ for all $n$. Assume that either

(i) $X$ or $A$ is an $\mathcal{L}_{p}$-space, or

(ii) $R_{n} B \subset B$ for all $n$, the operators $\left.R_{n}\right|_{B}$ are projections, and $X, A$ or $X / B$ is an $\mathcal{L}_{p}$-space.

Then there is an a.s. $\left\{P_{n}\right\}_{n=1}^{\infty}$ of $X \oplus W_{p}$ consisting of projections with $P_{n}\left(A \oplus W_{p}\right) \subset A \oplus W_{p}$.

If (ii) holds then in addition $\left.P_{n}\right|_{B}=\left.R_{n}\right|_{B}$ for all $n$.

Moreover, if $R_{n} R_{m}=R_{\min (m, n)}$ for some $m$ and $n$ then also

$$
P_{n} P_{m}=P_{\min (m, n)} \text {. }
$$


If $\left(R_{n} R_{m}-R_{\min (m, n)}\right) X \subset A$ for some $m$ and $n$ then also

$$
\left(P_{n} P_{m}-P_{\min (m, n)}\right)\left(X \oplus W_{p}\right) \subset A \oplus W_{p} .
$$

If either (i) holds and the $R_{n}-R_{n-1}$ define rank one operators on $X / A$, or (ii) holds and $\operatorname{dim}\left(R_{n}-R_{n-1}\right) B \leq 1$ for all $n$, then the operators $P_{n}-P_{n-1}$ induce rank one operators on $\left(X \oplus W_{p}\right) /\left(A \oplus W_{p}\right)$.

Proof. If $R_{n} B \subset B, n=1,2, \ldots$, then the $R_{n}$ define operators (called $R_{n}$ again) on $X / B$. Moreover, if the operators $\left.R_{n}\right|_{B}$ are projections then the map

$$
x+B \mapsto R_{n}\left(\mathrm{id}-R_{n}\right) x, \quad x \in X,
$$

makes sense, has norm $\leq\left\|R_{n}\left(\mathrm{id}-R_{n}\right)\right\|$ and will be called $R_{n}\left(\mathrm{id}-R_{n}\right)$ again.

Let $V$ be that space among $X, A$ or $X / B$ which is an $\mathcal{L}_{p^{-}}$-space. Fix $m_{n^{-}}$ dimensional subspaces $F_{n} \subset V$ with $\sup _{n} d\left(F_{n}, l_{p}^{m_{n}}\right)<\infty$ and $R_{n} V \subset F_{n}$. Put

$$
W= \begin{cases}\left(\sum_{n} \oplus F_{n}\right)_{(p)} & \text { if } 1 \leq p<\infty, \\ \left(\sum_{n} \oplus F_{n}\right)_{(0)} & \text { if } p=\infty .\end{cases}
$$

Then $W \sim W_{p}$. Now define $P_{n}: X \oplus W \rightarrow X \oplus W$ by

$$
\begin{aligned}
P_{n}\left(x,\left(f_{k}\right)\right)=((2 \mathrm{id}- & \left.R_{n}^{2}\right) R_{n}\left(R_{n} x+\left(\mathrm{id}-R_{n}^{2}\right) f_{n}\right), \\
& \left.\left(f_{1}, \ldots, f_{n-1},\left(\mathrm{id}-R_{n}^{2}\right)\left(R_{n} x+\left(\mathrm{id}-R_{n}^{2}\right) f_{n}\right), 0,0, \ldots\right)\right)
\end{aligned}
$$

for $x \in X, f_{k} \in F_{k}, k=1,2, \ldots$

(In the case $F_{n} \subset X / B$ take (id $\left.-R_{n}^{2}\right)\left(R_{n} x+B+\left(\mathrm{id}-R_{n}^{2}\right) f_{n}\right)$ in the $n$th component instead.)

The operators $P_{n}$ are of finite rank and we have $P_{n} \rightarrow$ id pointwise on $X \oplus W$. It is easily checked that the $P_{n}$ are projections, that $\left.R_{n}\right|_{B}=\left.P_{n}\right|_{B}$ if we are in case (ii), and that $P_{n} P_{m}=P_{\min (m, n)}$ provided that $R_{n} R_{m}=$ $R_{\min (m, n)}$.

Similarly, if $\left(R_{n} R_{m}-R_{\min (m, n)}\right) X \subset A$ for some $m$ and $n$ then $R_{n} R_{m}-$ $R_{\min (m, n)}$ induces the zero operator on $X / A$. We can easily check that then $P_{n} P_{m}-P_{\min (m, n)}$ induces the zero operator on $\left(X \oplus W_{p}\right) /\left(A \oplus W_{p}\right)$. Moreover, clearly $P_{n}(A \oplus W) \subset A \oplus W$ (since, by assumption, $R_{n}\left(\mathrm{id}-R_{n}\right) X$ $\subset A)$.

Finally, assume that the operators $R_{n}-R_{n-1}$ define rank one operators on $X / A$. By assumption, $R_{n}\left(\mathrm{id}-R_{n}\right) X \subset A$ and the operators on $X / A$ induced by $R_{n}$ and $\left(2 \mathrm{id}-R_{n}^{2}\right) R_{n}^{2}$ coincide. Hence, by definition of the $P_{n}$, the operators $P_{n}-P_{n-1}$ define rank one operators on $\left(X \oplus W_{p}\right) /\left(A \oplus W_{p}\right)$.

REMARK. The proof of 4.1 shows that actually

$$
\sup _{n}\left\|P_{n}\right\| \leq 18 \sup _{n}\left\|R_{n}\right\|^{4} .
$$


Proof of Theorem 2.1. If the operators $R_{n}$ define projections on $X / A$ or the operators $\left.R_{n}\right|_{B}$ are projections then $R_{n}\left(\mathrm{id}-R_{n}\right) X \subset A$ for all $n$. Hence Proposition 4.1 proves the FDD version of Theorem 2.1.

If the operators $R_{n}$ define basis projections on $X / A$ or on $B$ then $R_{n}-$ $R_{n-1}$ define rank one operators on $X / A$. Using Proposition 4.1 we find a sequence $\left\{P_{n}\right\}_{n=1}^{\infty}$ of FDD-projections of $X \oplus W_{p}$ with all the properties of 4.1 such that the operators $P_{n}-P_{n-1}$ induce rank one operators on $\left(X \oplus W_{p}\right) /\left(A \oplus W_{p}\right)$. This implies that there are subspaces $U_{n} \subset X \oplus W_{p}$ such that

$$
\left(P_{n}-P_{n-1}\right)\left(X \oplus W_{p}\right)=\left(P_{n}-P_{n-1}\right)\left(A \oplus W_{p}\right) \oplus U_{n}
$$

with $\operatorname{dim} U_{n} \leq 1$. Since the $P_{n}-P_{n-1}$ are uniformly bounded projections we find uniformly complemented subspaces $G_{n} \subset l_{p}^{m_{n}}$ for suitable $m_{n}$ such that

$$
\sup _{n} d\left(\left(P_{n}-P_{n-1}\right)\left(A \oplus W_{p}\right) \oplus G_{n}, l_{p}^{m_{n}}\right)<\infty .
$$

This is possible if any of $X, A$ or $X / B$ is an $\mathcal{L}_{p^{-}}$-space. (In the latter case we have $U_{n}=\left(P_{n}-P_{n-1}\right) B$. $)$

Since $\operatorname{dim}\left(P_{n}-P_{n-1}\right)\left(X \oplus W_{p}\right) /\left(P_{n}-P_{n-1}\right)\left(A \oplus W_{p}\right) \leq 1$ we also have

$$
\sup _{n} d\left(\left(P_{n}-P_{n-1}\right)\left(X \oplus W_{p}\right) \oplus G_{n}, l_{p}^{k_{n}}\right)<\infty \quad \text { for suitable } k_{n} .
$$

Put

$$
V_{p}= \begin{cases}\left(\sum_{n} \oplus G_{n}\right)_{(p)} & \text { if } 1 \leq p<\infty, \\ \left(\sum_{n} \oplus G_{n}\right)_{(0)} & \text { if } p=\infty .\end{cases}
$$

Then $V_{p}$ is complemented in $l_{p}$ or $c_{0}$, resp. Hence $V_{p} \sim l_{p}$ if $1 \leq p<\infty$, and $V_{\infty} \sim c_{0}([7])$. Put $Y_{p}=X \oplus W_{p} \oplus V_{p}$. Then $Y_{p} \sim X \oplus l_{p}$ if $1 \leq p<\infty$, and $Y_{\infty} \sim X \oplus c_{0}$. Define $\widehat{P}_{n}: Y_{p} \rightarrow Y_{p}$ by

$$
\widehat{P}_{n}\left(y,\left(g_{1}, g_{2}, \ldots\right)\right)=\left(P_{n} y,\left(g_{1}, \ldots, g_{n}, 0,0, \ldots\right)\right)
$$

for $y \in X \oplus W_{p}$ and $g_{k} \in G_{k}, k=1,2, \ldots$ Then the $\widehat{P}_{n}$ are FDD-projections on $Y_{p}$ with $\widehat{P}_{n}\left(A \oplus W_{p} \oplus V_{p}\right) \subset A \oplus W_{p} \oplus V_{p}$, and in the case of 2.1(b), $\left.\widehat{P}_{n}\right|_{B}=\left.R_{n}\right|_{B}$.

Finally, we have

$$
\left(\widehat{P}_{n}-\widehat{P}_{n-1}\right) Y_{p}=\left(P_{n}-P_{n-1}\right)\left(X \oplus W_{p}\right) \oplus G_{n}
$$

and $\operatorname{dim}\left(\widehat{P}_{n}-\widehat{P}_{n-1}\right) Y_{p} /\left(\widehat{P}_{n}-\widehat{P}_{n-1}\right)\left(A \oplus W_{p} \oplus V_{p}\right) \leq 1$. Since the summands $\left(\widehat{P}_{n}-\widehat{P}_{n-1}\right) Y_{p}$ are $l_{p}^{k_{n}}$-spaces they have bases with uniformly bounded basis constants, and suitable subsets are bases of $\left(\widehat{P}_{n}-\widehat{P}_{n-1}\right)\left(A \oplus W_{p} \oplus V_{p}\right)$. This shows that $Y_{p}$ has a basis with a suitable subsequence being a basis of $A \oplus W_{p} \oplus V_{p}$. Let $Q_{j}$ be the corresponding basis projections. In the case of $2.1(\mathrm{~b})$ we have $\operatorname{dim}\left(\widehat{P}_{n}-\widehat{P}_{n-1}\right) B \leq 1$ for all $n$. Hence for every 
$j$ there is $n$ such that $\left.Q_{j}\right|_{B}=\left.\widehat{P}_{n}\right|_{B}=\left.R_{n}\right|_{B}$. This completes the proof of $2.1(\mathrm{~b})$.

To prove 2.3 we need

4.2. Lemma. Let $X$ be an $\mathcal{L}_{p}$-space for some $p \in[1, \infty]$ and let $R_{k}$ : $X \rightarrow X, k=1, \ldots, n$, be linear, bounded and of finite rank. Then there is a finite rank projection $Q: X \rightarrow X$ with $R_{k} Q=Q R_{k}=R_{k}, k=1, \ldots, n$, where $\|Q\|$ does not depend on the operators $R_{k}$.

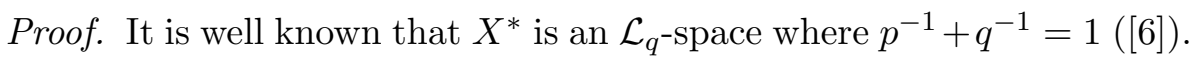
Since the $R_{k}^{*}: X^{*} \rightarrow X^{*}$ are of finite rank we find a finite rank projection $P: X^{*} \rightarrow X^{*}$ with $R_{k}^{*} X^{*} \subset P X^{*}$, where $\|P\|$ does not depend on the $R_{k}$. By [6, Corollary 3.2] we can choose $P$ to be $\mathrm{w}^{*}$-continuous. Regard $X$ as a natural subspace of $X^{* *}$. Then $Q_{1}=\left.P^{*}\right|_{X}$ is a projection with $Q_{1} X \subset X$. Since $P R_{k}^{*}=R_{k}^{*}$ we obtain $R_{k} Q_{1}=R_{k}$ for all $k$. Using the fact that $X$ is an $\mathcal{L}_{p}$-space we find a finite rank projection $Q_{2}: X \rightarrow X$ with $Q_{1} X \subset Q_{2} X$ and $R_{k} X \subset Q_{2} X, k=1, \ldots, n$, where $\left\|Q_{2}\right\|$ does not depend on the $R_{k}$. Hence we obtain $Q_{2} R_{k}=R_{k}, k=1, \ldots, n$, and $Q_{2} Q_{1}=Q_{1}$. Put $Q=Q_{1}+\left(\right.$ id $\left.-Q_{1}\right) Q_{2}$. Then $Q$ is a finite rank projection satisfying $R_{k} Q=Q R_{k}=R_{k}, k=1, \ldots, n$, and $\|Q\|$ does not depend on the $R_{k}$.

Proof of Theorem 2.3. Let $\left\{R_{n}\right\}_{n=1}^{\infty}$ be an a.s. of $X$ such that $R_{n} A \subset A$ for all $n$ and the operators $R_{n}$ define FDD-projections $\widehat{R}_{n}$ on $X / A$. Moreover, let $X$ be an $\mathcal{L}_{1}$-space. We prove the theorem in two steps.

(a) First we construct an a.s. $\left\{P_{n}\right\}_{n=1}^{\infty}$ of $X \oplus l_{1}$ such that $P_{n}\left(A \oplus l_{1}\right) \subset$ $A \oplus l_{1}$ for all $n$, the $P_{n}$ define FDD-projections on $\left(X \oplus l_{1}\right) /\left(A \oplus l_{1}\right)$, and $P_{m} P_{n}=P_{m}$ whenever $m \leq n$. Moreover, $P_{n}-P_{n-1}$ shall induce rank one operators on $\left(X \oplus l_{1}\right) /\left(A \oplus l_{1}\right)$ if $\operatorname{dim}\left(\widehat{R}_{n}-\widehat{R}_{n-1}\right)(X / A) \leq 1$ for all $n$.

Indeed, by Proposition 4.1 we may assume that the $R_{n}$ are already projections. Fix uniformly bounded finite rank projections $Q_{n}: X \rightarrow X$ with $R_{j} Q_{n}=R_{j}=Q_{n} R_{j}$ and $Q_{j} Q_{n}=Q_{n}=Q_{n} Q_{j}, j=1, \ldots, n$, which exist according to 4.2. Consider subspaces $F_{n} \subset X$ with $Q_{n} X \subset F_{n}$ and $\sup _{n} d\left(F_{n}, l_{1}^{m_{n}}\right)<\infty$ for some $m_{n}$. Then $F:=\left(\sum_{n} \oplus F_{n}\right)_{(1)} \sim l_{1}$. Now define $P_{n}: X \oplus F \rightarrow X \oplus F$ by

$$
\begin{aligned}
P_{n}\left(x,\left(f_{1}, f_{2}, \ldots\right)\right) & =\left(R_{n}\left(x+\sum_{k=n}^{\infty}\left(\mathrm{id}-R_{k}\right) f_{k}\right),\right. \\
& \left.\left(f_{1}, \ldots, f_{n-1},\left(Q_{n}-R_{n}\right)\left(x+\sum_{k=n}^{\infty}\left(\mathrm{id}-R_{k}\right) f_{k}\right), 0, \ldots 0\right)\right) .
\end{aligned}
$$

If $x \in A$ then clearly $P_{n}\left(x,\left(f_{k}\right)\right) \subset A \oplus F$ since $R_{n}\left(\mathrm{id}-R_{k}\right) X \subset A$ for all $k \geq n$. Moreover, $R_{n}^{2}=R_{n}$ and $\left(Q_{n}-R_{n}\right)^{2}=Q_{n}-R_{n}$. This implies that $P_{m} P_{n}=P_{m}$ whenever $m \leq n$. If $j \geq n$ then we easily see that 


$$
\begin{aligned}
&\left(P_{j} P_{n}-P_{n}\right)\left(x,\left(f_{k}\right)\right)=\left(\left(R_{j}-\mathrm{id}\right) R_{n}\left(x+\sum_{k=n}^{\infty}\left(\mathrm{id}-R_{k}\right) f_{k}\right),\right. \\
&\left.(\underbrace{0, \ldots, 0}_{j-1},\left(Q_{j}-R_{j}\right) R_{n}\left(x+\sum_{k=n}^{\infty}\left(\mathrm{id}-R_{k}\right) f_{k}\right), 0, \ldots)\right) \in A \oplus F .
\end{aligned}
$$

This implies $\left(P_{m} P_{n}-P_{\min (m, n)}\right)\left(X \oplus l_{1}\right) \subset A \oplus F$ and hence the $P_{n}$ define FDD-projections on $\left(X \oplus l_{1}\right) /\left(A \oplus l_{1}\right)$.

Finally, since the $R_{n}$ are projections, we have

$$
\begin{aligned}
\left(P_{n}-P_{n-1}\right)\left(x,\left(f_{k}\right)\right) & =\left(\left(R_{n}-R_{n-1}\right)\left(x+\sum_{k=n}^{\infty}\left(\mathrm{id}-R_{k}\right) f_{k}\right),\right. \\
& (\underbrace{0, \ldots, 0}_{n-2}, f_{n-1}-\left(Q_{n-1}-R_{n-1}\right)\left(x+\sum_{k=n-1}^{\infty}\left(\mathrm{id}-R_{k}\right) f_{k}\right), \\
& \left.\left.\left(Q_{n}-R_{n}\right)\left(x+\sum_{k=n}^{\infty}\left(\mathrm{id}-R_{k}\right) f_{k}\right), 0, \ldots\right)\right) .
\end{aligned}
$$

Since $\left(R_{n}-R_{n-1}\right) \sum_{k=n}^{\infty}\left(\mathrm{id}-R_{k}\right) f_{k} \in A$, the $P_{n}-P_{n-1}$ define rank one operators on $\left(X \oplus l_{1}\right) /\left(A \oplus l_{1}\right)$ provided that $\operatorname{dim}\left(\widehat{R}_{n}-\widehat{R}_{n-1}\right)(X / A) \leq 1$ for all $n$.

(b) Now we prove 2.3. According to (a), taking $X \oplus l_{1}$ instead of $X$ and $A \oplus l_{1}$ instead of $A$, we can assume that $\left\{R_{n}\right\}_{n=1}^{\infty}$ is an a.s. of $X$ with $R_{n} A \subset A, R_{m} R_{n}=R_{m}$ for $m \leq n$ such that the $R_{n}$ define FDD-projections $\widehat{R}_{n}$ on $X / A$. Since $R_{n} \rightarrow$ id and the $R_{n}$ are of finite rank, using a perturbation argument, we may as well assume that there is a subsequence $\left\{R_{n_{m}}\right\}_{m=1}^{\infty}$ which is a c.a.s. of $X$. Then the operators $R_{n_{m}}$ are FDD-projections. This proves the FDD-part of $2.2(\mathrm{a})$.

Now assume that in addition $\operatorname{dim}\left(\widehat{R}_{n}-\widehat{R}_{n-1}\right)(X / A) \leq 1$ for all $n$. Put $X_{m}=\left(R_{n_{m}}-R_{n_{m-1}}\right) X$ and $A_{m}=\left(R_{n_{m}}-R_{n_{m-1}}\right) A$. We may assume that $R_{j} X_{m} \subset X_{m}$ for all $j=n_{m-1}+1, \ldots, n_{m}$. (Otherwise replace $R_{j}$ by $R_{n_{m-1}}+\left(R_{n_{m}}-R_{n_{m-1}}\right) R_{j}\left(R_{n_{m}}-R_{n_{m-1}}\right)$.) Find subspaces $G_{m} \subset X$ with $X_{m} \subset G_{m}$ and $\sup _{m} d\left(G_{m}, l_{1}^{k_{m}}\right)<\infty$ for some $k_{m}$. Put

$$
Y_{m}=(\underbrace{G_{m} \oplus \ldots \oplus G_{m}}_{n_{m}-n_{m-1} \text { times }})_{(1)} \text {. }
$$

Then $Z:=X \oplus\left(\sum_{m} \oplus Y_{m}\right)_{(1)} \sim X \oplus l_{1}$. Moreover, the $X_{m} \oplus Y_{m}$ are the summands of an FDD of $Z$. Let $P_{n_{m}}$ be the corresponding projections and put $W=A \oplus\left(\sum_{m} \oplus Y_{m}\right)_{(1)}$. Then $P_{n_{m}} W \subset W$ for all $m$. We complete the $P_{n_{m}}$ to a c.a.s. $\left\{P_{j}\right\}_{j=1}^{\infty}$ of $Z$ with $P_{j} W \subset W$ such that the $P_{j}$ define basis projections on $Z / W$. Then an application of 2.1(a) finishes the proof. 
To this end put $M=n_{m}-n_{m-1}$ and define $\bar{P}_{l}: X_{m} \oplus Y_{m} \rightarrow X_{m} \oplus Y_{m}$ by

$$
\begin{aligned}
\bar{P}_{l}\left(x,\left(g_{1}, \ldots, g_{M}\right)\right)= & \left(R_{l} x-\left(\mathrm{id}-R_{l}\right) \sum_{k=1}^{l-1-n_{m-1}} R_{k+n_{m-1}} g_{k},\right. \\
& \left.\left(g_{1}, \ldots, g_{l-1},-R_{l}\left(x+\sum_{k=1}^{l-1-n_{m-1}} R_{k+n_{m-1}} g_{k}\right), 0, \ldots, 0\right)\right)
\end{aligned}
$$

for $l=n_{m-1}+1, \ldots, n_{m}$. Then the $\bar{P}_{l}$ are uniformly bounded and $\bar{P}_{l} \bar{P}_{k}=$ $\bar{P}_{\min (k, l)}$ for all $k$ and $l$. Moreover, $\bar{P}_{l}\left(A_{m} \oplus Y_{m}\right) \subset A_{m} \oplus Y_{m}$ since (id $\left.-R_{l}\right) R_{h} X \subset A$ if $l \geq h$. From the latter fact we also infer that the $\bar{P}_{l}-\bar{P}_{l-1}$ define rank one operators on $\left(X_{m} \oplus Y_{m}\right) /\left(A_{m} \oplus Y_{m}\right)$. Now, finally, put

$P_{l}=P_{n_{m}}+\left(P_{n_{m}}-P_{n_{m-1}}\right) \bar{P}_{l}\left(P_{n_{m}}-P_{n_{m-1}}\right), \quad n_{m-1}+1 \leq l \leq n_{m}, l=1,2, \ldots$

Then the $P_{l}$ satisfy all the requirements to apply $2.1(\mathrm{a})$.

To prove Theorem 2.4, let $X$ be an $\mathcal{L}_{\infty}$-space, $A \subset X$ a closed subspace, and $\left\{R_{k}\right\}_{k=1}^{\infty}$ an a.s. of $X$ such that $R_{n} A \subset A$ for all $n$ and the operators $R_{n}$ induce basis projections on $X / A$. By Proposition 4.1 we may assume that the operators $R_{k}$ are projections.

4.3. Lemma. Put $X_{1}=X \oplus c_{0}$ and $A_{1}=A \oplus c_{0}$. Then there is a closed subspace $B \subset X_{1}$ such that

$$
B \sim c_{0}, \quad A_{1} \cap B=\{0\}, \quad \overline{A_{1}+B}=X_{1} .
$$

Furthermore, there exists an a.s. $\left\{S_{k}\right\}_{k=1}^{\infty}$ of $X_{1}$ consisting of projections such that

$$
\left.S_{n}\right|_{X}=R_{n} \quad \text { for all } n,
$$

$A_{1}$ and $B$ are invariant under $S_{n}$ and the operators $\left.S_{n}\right|_{B}$ are the projections of the unit vector basis of $c_{0}$. Moreover,

$$
S_{m}\left(\mathrm{id}-S_{n}\right) X_{1} \subset A, \quad\left(\mathrm{id}-S_{n}\right) S_{m} X_{1} \subset A \quad \text { whenever } m \leq n .
$$

Finally, there is a bounded projection $Q: X_{1} \rightarrow B$ with

$$
X=\operatorname{ker} Q
$$

(where we identify $x \in X$ with $\left.(x, 0) \in X_{1}\right)$.

Proof. Find $x_{n} \in X$ such that $\left\|x_{n}\right\| \leq 2$ and the elements $x_{n}+A$ are the elements of a normalized basis of $X / A$ whose basis projections are induced by the operators $R_{n}$. This implies

$$
R_{k} x_{n} \in A \quad \text { whenever } k<n, \quad\left(\mathrm{id}-R_{k}\right) x_{n} \in A \quad \text { whenever } k>n .
$$


Let $\left\{e_{k}\right\}_{k=1}^{\infty}$ be the unit vector basis of $c_{0}$. Put $b_{n}=\left(2^{-n} x_{n}, e_{n}\right) \in X_{1}$ and let $B=\overline{\operatorname{span}}\left\{b_{n}\right\}_{n=1}^{\infty} \subset X_{1}$. Define $Q: X_{1} \rightarrow B$ by

$$
Q\left(x, \sum_{k} \alpha_{k} e_{k}\right)=\left(\sum_{k} \frac{1}{2^{k}} \alpha_{k} x_{k}, \sum_{k} \alpha_{k} e_{k}\right) .
$$

Then clearly (4.1) is satisfied since $\left\{b_{n}\right\}_{n=1}^{\infty}$ is equivalent to the unit vector basis of $c_{0}$. We also obtain (4.4).

Now define $S_{k}: X_{1} \rightarrow X_{1}$ by $S_{k}(x, 0)=\left(R_{k} x, 0\right)$ for $x \in X$, and

$$
\begin{aligned}
& S_{k}\left(0, \sum_{n=1}^{\infty} \alpha_{n} e_{n}\right) \\
& \quad=\left(-\sum_{n=k+1}^{\infty} \frac{\alpha_{n}}{2^{n}} R_{k} x_{n}+\sum_{n=1}^{k} \frac{\alpha_{n}}{2^{n}}\left(\mathrm{id}-R_{k}\right) x_{n}, \sum_{n=1}^{k} \alpha_{n} e_{n}\right) .
\end{aligned}
$$

This implies in particular that $\left\{S_{k}\right\}_{k=1}^{\infty}$ is an a.s. of $X_{1}$ and that $S_{k} A_{1} \subset A_{1}$ for all $k$ (see (4.5)). Furthermore, we have

$$
S_{k} b_{n}= \begin{cases}b_{n} & \text { if } k \geq n, \\ 0 & \text { if } k<n .\end{cases}
$$

This shows that the $S_{k}$ are projections (since the $R_{k}$ are assumed to be projections). We obtain (4.2).

We have $S_{k}\left(\mathrm{id}-S_{m}\right) X=R_{k}\left(\mathrm{id}-R_{m}\right) X \subset A$ whenever $k \leq m$ by assumption on $X / A$. Similarly (id $\left.-S_{m}\right) S_{k} X \subset A$ if $k \leq m$. Using (4.6) we see that, for $k \leq m$ and any $n$,

$$
\begin{aligned}
S_{k}\left(\mathrm{id}-S_{m}\right)\left(0, e_{n}\right) & =S_{k}\left(\mathrm{id}-S_{m}\right)\left(b_{n}-\left(\frac{x_{n}}{2^{n}}, 0\right)\right) \\
& =\left(-\frac{1}{2^{n}} R_{k}\left(\mathrm{id}-R_{m}\right) x_{n}, 0\right) \in A,
\end{aligned}
$$

and similarly,

$$
\left(\mathrm{id}-S_{m}\right) S_{k}\left(0, e_{n}\right)=\left(-\frac{1}{2^{n}}\left(\mathrm{id}-R_{m}\right) R_{k} x_{n}, 0\right) \in A .
$$

Hence we obtain (4.3), which completes the proof of Lemma 4.3.

Remark. (4.4) implies that $X \oplus B=X_{1}$. This means in particular that $S_{m} S_{n}=S_{\min (m, n)}$ whenever $R_{m} R_{n}=R_{\min (m, n)}$.

We retain the notation of Lemma 4.3. Consider the unit vector basis $\left\{b_{n}\right\}_{n=1}^{\infty}$ of $B \sim c_{0}$. The projections $S_{k}$ induce the basis projections for the basis $\left\{b_{n}+A_{1}\right\}_{n=1}^{\infty}$ of $X_{1} / A_{1}$. Hence we find $b_{n}^{*} \in A_{1}^{\perp} \subset X_{1}^{*}$ with

$$
b_{n}^{*}\left(b_{m}\right)= \begin{cases}1, & n \neq m \\ 0, & n=m\end{cases}
$$

(The functionals $b_{n}^{*}$ may not be uniformly bounded.) 
Let $\widetilde{Q}_{n}: X_{1} \rightarrow \operatorname{span}\left\{b_{n}\right\}$ be defined by $\widetilde{Q}_{n} x=b_{n}^{*}(x) b_{n}$. Then

$$
S_{m} \widetilde{Q}_{n}=\widetilde{Q}_{n} S_{m} \quad \text { for all } n, m, \quad \widetilde{Q}_{n} S_{m}= \begin{cases}\widetilde{Q}_{n}, & n \leq m, \\ 0, & m<n .\end{cases}
$$

Moreover,

$$
S_{n} x-\sum_{k=1}^{n} \widetilde{Q}_{k} x \in A_{1} \quad \text { for any } n \text { and any } x \in X_{1} .
$$

( $\sum_{k=1}^{n} \widetilde{Q}_{k} x$ is the projection of $S_{n} x$ onto $B$ along $A_{1}$.) Now (4.3) implies (4.10) $\quad \widetilde{Q}_{n} S_{m}\left(\mathrm{id}-S_{n}\right)=0, \quad \widetilde{Q}_{n}\left(\mathrm{id}-S_{n}\right) S_{m}=0 \quad$ whenever $m \leq n$.

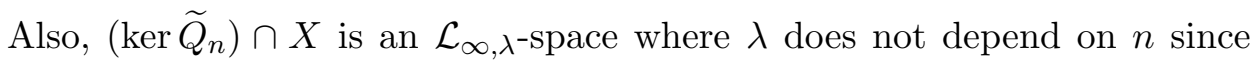
this space is 1-codimensional in $X$.

4.4. Proposition. There is an a.s. $\left\{T_{n}\right\}_{n=1}^{\infty}$ of $X_{1} \oplus c_{0}$ consisting of projections and leaving $A_{1} \oplus c_{0}$ and $B$ invariant such that

$$
T_{m} T_{n}=T_{m} \quad \text { whenever } m \leq n,\left.\quad T_{k}\right|_{B}=\left.S_{k}\right|_{B} \quad \text { for all } k .
$$

(We identify $x \in X_{1}$ with $(x, 0) \in X_{1} \oplus c_{0}$.)

Proof. Find finite-dimensional subspaces $F_{n} \subset \operatorname{ker} \widetilde{Q}_{n} \cap X$ with

$$
\bigcup_{k=n}^{\infty} S_{n}\left(\mathrm{id}-S_{k}\right) X_{1} \subset F_{n}, \quad \bigcup_{m=1}^{n}\left(\mathrm{id}-\widetilde{Q}_{n}\right) S_{m} X \subset F_{n}
$$

and $\sup _{n} d\left(F_{n}, l_{\infty}^{m_{n}}\right)<\infty$ where $m_{n}=\operatorname{dim} F_{n}$. Put $G_{n}=F_{n}+\operatorname{span}\left\{b_{n}\right\}$. Hence $\sup _{n} d\left(G_{n}, l_{\infty}^{m_{n}+1}\right)<\infty$. Note that with the projection $Q: X_{1} \rightarrow B$ of Lemma 4.3, using (4.4) we have

$$
Q G_{n}=\operatorname{span}\left\{b_{n}\right\}, \quad \operatorname{ker} Q \cap G_{n}=F_{n} .
$$

Put $X_{2}=X_{1} \oplus\left(\sum_{n} \oplus G_{n}\right)_{(0)}$. Hence $X_{2} \sim X_{1} \oplus c_{0}$. Define $T_{n}: X_{2} \rightarrow X_{2}$ by

$$
\begin{aligned}
& T_{n}\left(x,\left(g_{1}, g_{2}, \ldots\right)\right)=\left(S_{n}\left(x+g_{n}\right),\left(g_{1}+S_{1}\left(\mathrm{id}-S_{n}\right) x-S_{1} S_{n} g_{n},\right.\right. \\
&\left.\left.\ldots, g_{n-1}+S_{n-1}\left(\mathrm{id}-S_{n}\right) x-S_{n-1} S_{n} g_{n}, 0,0, \ldots\right)\right) .
\end{aligned}
$$

The definition of $T_{n}$ makes sense since we have $S_{m}\left(\mathrm{id}-S_{n}\right) X_{1} \subset F_{m}$ and $S_{m} S_{n} G_{n} \subset G_{m}$ for $m \leq n$. The latter inclusion follows from the fact that $S_{m} S_{n} b_{n}=0$ if $m<n$ and $S_{m} S_{n} F_{n} \subset G_{m}$ (in view of (4.12)). The $T_{n}$ are uniformly bounded projections and $\left.T_{n}\right|_{B}=\left.S_{n}\right|_{B}$ since $\left.S_{m}\left(\mathrm{id}-S_{n}\right)\right|_{B}=0$ for $m \leq n$. We easily check that (4.11) is satisfied. Put

$$
b(n)=\left(b_{n},(\underbrace{0, \ldots, 0}_{n-1},-b_{n}, 0,0, \ldots)\right) \in X_{2}
$$

and $W=\overline{\operatorname{span}}\{b(n)\}_{n=1}^{\infty}$. Moreover, put $V=W+\left(\sum_{n} \oplus F_{n}\right)_{(0)}$. Then, in 
view of (4.13), we have $V \sim c_{0}$ and $X_{2} \sim X_{1} \oplus V$. Also, (4.14) implies

$$
T_{n} b(m)= \begin{cases}b(m), & m<n, \\ 0, & n \leq m\end{cases}
$$

(Recall that $S_{n} b_{m}=0$ if $m>n$, since the operators $\left.S_{n}\right|_{B}$ are the basis projections of $\left.\left\{b_{k}\right\}_{k=1}^{\infty}.\right)$ Moreover, if $f \in F_{m}$ and $f(m)=(0,(\underbrace{0, \ldots, 0}_{m-1}, f, 0,0, \ldots))$,

and

$$
T_{n} f(m)= \begin{cases}f(m), & m<n \\ 0, & m>n\end{cases}
$$

$$
\begin{aligned}
T_{n} f(n)+V=( & S_{n} f-\sum_{k=1}^{n} \widetilde{Q}_{k} f, \\
& \left.\left(-S_{1} S_{n} f+\widetilde{Q}_{1} f, \ldots,-S_{n-1} S_{n} f+\widetilde{Q}_{n-1} f, 0,0, \ldots\right)\right) .
\end{aligned}
$$

(Recall that $\widetilde{Q}_{n} f=0$ if $f \in F_{n} \subset \operatorname{ker} \widetilde{Q}_{n} \cap X$.) In view of (4.8) and (4.9) this implies $T_{n} f(n) \in A_{1}+V$. On the other hand, if $a \in A_{1}$, then

$$
T_{n}(a,(0,0, \ldots)) \in A_{1}+\left(\sum_{k} \oplus F_{k}\right)_{(0)}
$$

according to (4.12) and (4.14). Hence $T_{n}\left(A_{1}+V\right) \subset A_{1}+V$ for all $n$. We clearly have, in view of (4.13), $A_{1}+V \sim A_{1} \oplus c_{0}$.

4.5. Proposition. There is an a.s. $\left\{P_{n}\right\}_{n=1}^{\infty}$ of $X_{1} \oplus c_{0}$ consisting of projections and leaving $A_{1} \oplus c_{0}$ and $B$ invariant such that

$$
P_{n} P_{m}=P_{m} \quad \text { whenever } m \leq n,\left.\quad P_{k}\right|_{B}=\left.S_{k}\right|_{B} \quad \text { for all } k .
$$

Moreover,

$$
P_{m} P_{n}=P_{m} \quad \text { whenever } R_{m} R_{n}=R_{m} \text { and } m \leq n .
$$

Proof. Since $\lim _{k \rightarrow \infty} S_{k} x=x$ for all $x \in X_{1}$ the space $\operatorname{span}\left(\bigcup_{k} S_{k} X_{1}\right)$ is dense in $X_{1}$. Find finite-dimensional subspaces

$$
F_{n} \subset \operatorname{span}\left(\bigcup_{k} S_{k} X_{1}\right) \cap \operatorname{ker} \widetilde{Q}_{n+1} \cap X
$$

with

$$
\bigcup_{m=1}^{n}\left(\mathrm{id}-S_{n}\right) S_{m} X_{1} \subset F_{n}, \quad \bigcup_{m=1}^{n} S_{m} X \subset F_{n} .
$$

This is possible, since by (4.3),

$$
\left(\mathrm{id}-S_{n}\right) S_{m} X_{1} \subset A \cap \operatorname{span}\left(\bigcup_{k} S_{k} X_{1}\right) \subset X \cap \operatorname{span}\left(\bigcup_{k} S_{k} X\right),
$$

and by (4.8), $\widetilde{Q}_{n+1}\left(\bigcup_{m=1}^{n} S_{m} X\right)=0$. (Recall that $S_{k} X \subset X$ for all $k$ in view 
of (4.2).) Finally, $F_{n}$ can be arranged such that in addition $\sup _{n} d\left(F_{n}, l_{\infty}^{\operatorname{dim} F_{n}}\right)$ $<\infty$, since $X \cap \operatorname{ker} \widetilde{Q}_{n+1}$ is an $\mathcal{L}_{\infty}$-space. Put $G_{n}=F_{n}+\operatorname{span}\left\{b_{n+1}\right\}$. Hence $\sup _{n} d\left(G_{n}, l_{\infty}^{\operatorname{dim} G_{n}}\right)<\infty$. We have

$$
Q G_{n}=\operatorname{span}\left\{b_{n+1}\right\}, \quad \operatorname{ker} Q \cap G_{n}=F_{n},
$$

in view of (4.4) since $F_{n} \subset X$.

Now put $X_{2}=X_{1} \oplus\left(\sum_{n} \oplus G_{n}\right)_{(0)} \sim X_{1} \oplus c_{0}$. Define $P_{n}: X_{2} \rightarrow X_{2}$ by

$$
\begin{aligned}
& P_{n}\left(x,\left(g_{1}, g_{2}, \ldots\right)\right)=\left(S_{n} x+\left(\mathrm{id}-S_{n}\right) g_{n},\left(g_{1}, \ldots, g_{n},\right.\right. \\
& \left.\left.\left(\mathrm{id}-S_{n+1}\right)\left(S_{n} x+\left(\mathrm{id}-S_{n}\right) g_{n}\right),\left(\mathrm{id}-S_{n+2}\right)\left(S_{n} x+\left(\mathrm{id}-S_{n}\right) g_{n}\right), \ldots\right)\right) .
\end{aligned}
$$

The definition of $P_{n}$ makes sense in view of (4.18). In particular we have $\left(\mathrm{id}-S_{n+k}\right)\left(\mathrm{id}-S_{n}\right) G_{n} \subset G_{n+k}$ since $\left(\mathrm{id}-S_{n+k}\right)\left(\mathrm{id}-S_{n}\right) b_{n+1}=0$ for $k \geq 1$. The operators $P_{n}$ are uniformly bounded projections. We obtain $\left.P_{n}\right|_{B}=$ $\left.S_{n}\right|_{B}$ since (id $\left.-S_{n+k}\right) S_{n} B=\{0\}$. It is easily checked that $P_{n} P_{m}=P_{m}$ whenever $n \geq m$. If $R_{m} R_{n}=R_{m}$ and $m \leq n$ then, in view of $X \oplus B=X_{1}$, by (4.2) we see that $S_{m} S_{n}=S_{m}$. Also, (4.20) implies that then $P_{m} P_{n}=P_{m}$.

Put

$$
b(n)=\left(b_{n+1},(\underbrace{0, \ldots, 0}_{n-1}, b_{n+1}, 0,0, \ldots)\right) \in X_{2}
$$

and $W=\overline{\operatorname{span}}\{b(n)\}_{n=1}^{\infty}$. Moreover, put $V=W+\left(\sum_{n} \oplus F_{n}\right)_{(0)}$. Then, in view of (4.19) and the fact that $\left\{b_{n}\right\}_{n=1}^{\infty}$ is the unit vector basis of $c_{0}$, we have $X_{2}=X_{1} \oplus V \sim X_{1} \oplus c_{0}$. Equation (4.20) implies

$$
P_{n} b(m)= \begin{cases}b(m), & m \leq n, \\ 0, & m>n .\end{cases}
$$

(This follows since $S_{n} b_{m}$ is $b_{m}$ if $m \leq n$, and 0 otherwise.)

Finally, take $f \in F_{m}$ and put

Then (4.20) implies

$$
f(m)=(0,(\underbrace{0, \ldots, 0}_{m-1}, f, 0,0, \ldots))
$$

and

$$
P_{n} f(m)= \begin{cases}f(m), & m<n, \\ 0, & m>n\end{cases}
$$

$$
\begin{aligned}
& P_{n} f(n)+V=\left(\left(\mathrm{id}-S_{n}\right) f-\sum_{k=n+1}^{\infty} \widetilde{Q}_{k} f,(\underbrace{0, \ldots, 0}_{n-1}, f\right. \\
& \left.\left.\quad\left(\mathrm{id}-S_{n+1}\right)\left(\mathrm{id}-S_{n}\right) f-\widetilde{Q}_{n+2} f,\left(\mathrm{id}-S_{n+2}\right)\left(\mathrm{id}-S_{n}\right) f-\widetilde{Q}_{n+3} f, \ldots\right)\right) .
\end{aligned}
$$

Note that, in view of (4.17), $\widetilde{Q}_{n+k} f=0$ for some $k_{0}$ and all $k \geq k_{0}$, and also $\widetilde{Q}_{n+1} f=0$. In particular, there is no problem with the convergence of the series. Now, (id $\left.-S_{n}\right) f-\sum_{k=n+1}^{\infty} \widetilde{Q}_{k} f$ is the projection of $\left(\right.$ id $\left.-S_{n}\right) f$ onto 
$A_{1}$ along $B$. Moreover, by (4.8), we have

$$
\widetilde{Q}_{n+k+1}\left(\mathrm{id}-S_{n+k}\right)\left(\mathrm{id}-S_{n}\right) f=\widetilde{Q}_{n+k+1} f .
$$

Hence $P_{n} V \subset A_{1}+V$. In view of (4.3) and (4.20) we also have $P_{n} A_{1} \subset$ $A_{1}+V$. Since $A_{1}+V \sim A \oplus c_{0}$ the proof is complete.

Proof of Theorem 2.4. First we apply Lemma 4.3 to obtain $X_{1}=X \oplus c_{0}$, $A_{1}=A \oplus c_{0}$ and $S_{k}$. Then we continue with Proposition 4.4 to obtain

$$
X_{2}=X_{1} \oplus c_{0} \sim X \oplus c_{0}, \quad A_{2} \sim A_{1} \oplus c_{0} \sim A \oplus c_{0}
$$

and $T_{k}$.

Then we apply Lemma 4.3 and the remark following it again to $X_{2}$ instead of $X, A_{2}$ instead of $A$, and $T_{k}$ instead of $R_{k}$. Finally, we apply Proposition 4.5 to find $P_{n}$ on $X \oplus c_{0}$. Since $T_{m} T_{n}=T_{m}$ for $m \leq n$, by (4.16) we also have $P_{m} P_{n}=P_{m}$. Hence $\left\{P_{n}\right\}_{n=1}^{\infty}$ is a sequence of FDD-projections on $X \oplus c_{0}$ leaving $A \oplus c_{0}$ invariant. Moreover, there is a subspace $B \subset X \oplus c_{0}$ with

$$
\overline{\left(A \oplus c_{0}\right)+B}=X \oplus c_{0}, \quad B \sim c_{0}
$$

such that the operators $\left.P_{n}\right|_{B}$ are the projections of the unit vector basis of $c_{0}$. Now an application of Theorem 2.1(b) (and the following remark) finishes the proof.

\section{References}

[1] P. G. Casazza, Approximation properties, in: Handbook of the Geometry of Banach Spaces, Vol. I, W. B. Johnson and J. Lindenstrauss (eds.), North-Holland, Amsterdam, 2001, 271-316.

[2] R. E. Edwards, Fourier Series, Vol. 2, Springer, Berlin, 1982.

[3] K. Hoffman, Banach Spaces of Analytic Functions, Prentice-Hall, Englewood Cliffs, NJ, 1962.

[4] W. B. Johnson, H. P. Rosenthal and M. Zippin, On bases, finite dimensional decompositions and weaker structures in Banach spaces, Israel J. Math. 9 (1971), 488-506.

[5] N. J. Kalton and A. Pełczyński, Kernels of surjections from $\mathcal{L}_{1}$-spaces with an application to Sidon sets, Math. Ann. 309 (1997), 135-158.

[6] J. Lindenstrauss and H. P. Rosenthal, The $\mathcal{L}_{p}$ spaces, Israel J. Math. 7 (1969), $325-349$.

[7] J. Lindenstrauss and L. Tzafriri, Classical Banach Spaces I, Springer, Berlin, 1977.

[8] W. Lusky, On Banach spaces with bases, J. Funct. Anal. 138 (1996), 410-425.

[9] —, Three space properties and basis extensions, Israel J. Math. 107 (1998), 17-27.

[10] A. Pełczyński, Any separable Banach space with the bounded approximation property is a complemented subspace of a Banach space with a basis, Studia Math. 40 (1971), 239-242.

[11] C. J. Read, Different forms of the approximation property, to appear. 
[12] S. J. Szarek, A Banach space without a basis which has the bounded approximation property, Acta Math. 159 (1987), 81-98.

Institute for Mathematics

University of Paderborn

Warburger Str. 100

D-33098 Paderborn, Germany

E-mail: lusky@math-mail.uni-paderborn.de

Received December 18, 2002

Revised version May 30, 2003 